Recent Insights into the Physics of the Sun and Heliosphere:

Highlights from $\mathrm{SOHO}$ and Other Space Missions

IAU Symposium, Vol. 203, 2001

P. Brekke, B. Fleck, and J. B. Gurman eds.

\title{
Components of the Local Interstellar Medium
}

\author{
J. L. Linsky, S. Redfield \& B. Wood \\ JILA, University of Colorado, Boulder, CO 80309-0440 USA
}

\begin{abstract}
HST, EUVE, and Ca II spectra are providing critical velocity and column density data needed to identify individual stuctures (clouds) of warm gas in the local ISM. The Sun is located very close to the edge of and will soon leave the Local Interstellar Cloud (LIC). We will summarize the properties of the LIC and other nearby warm clouds.
\end{abstract}

The heliosphere is moving through warm partially ionized interstellar gas that forms the outer boundary of the solar wind, constraining its size and properties. Since HST's launch in 1990, we have exploited the high spectral resolution and excellent photometric qualities of its two spectrographs (GHRS and STIS) to study the interstellar medium along lines of sight toward nearby late-type stars. Our objectives have been to measure the $\mathrm{D} / \mathrm{H}$ ratio and its spatial variations, to study the interaction of solar and stellar winds with the interstellar gas, and to determine the physical properties and structure of warm clouds near the Sun. The first topic was reviewed by Linsky (1998), and the second by Wood, Linsky, \& Zank (2000) and by Wood, Müller, \& Zank (2000). In this talk, we summarize what we have learned about the nearby warm clouds.

High resolution ultraviolet spectroscopy permits us to measure the column densities and broadening parameters for resonance lines of many atoms and ions including the Lyman $\alpha(\mathrm{L} \alpha)$ lines of H I $(1215.67 \AA)$ and D I (1215.34 $\AA)$. Absorption in the H I line has three components: (1) highly saturated absorption by interstellar hydrogen, (2) additional absorption to the red of the ISM component due to the pileup of neutral hydrogen in the "hydrogen wall" (cf. Baranov \& Malama 1995; Zank 1999) near the heliopause due to charge exchange between inflowing interstellar hydrogen and outflowing solar wind protons, and (3) additional absorption to the blue of the ISM component due to $\mathrm{H} \mathrm{I}$ in the stellar hydrogen wall where interstellar gas interacts with the wind of the cool star observed by HST. Astropause absorption is a new technique and perhaps the only one capable of measuring properties of the winds of solar-like stars.

Many studies (e.g., Linsky et al. (1995) and Wood et al. (1996)) use nearby late-type stars as background sources. In these studies the intrinsic stellar $\mathrm{L} \alpha$ emission line must be assumed or reconstructed (for stars with high or variable radial velocities). Narrow interstellar absorption lines of heavy elements like Mg II $(2796,2803 \AA)$, Fe II (2599 $\AA)$, and C II (1335 $\AA)$ provide important information on the turbulent (nonthermal) broadening parameter $\xi$ and separate individual velocity components produced by separate clouds.

We define a "cloud" as a structure characterized by a single bulk velocity vector, $T, \xi$, and metal depletion. Lallement \& Bertin (1992) and Lallement et al. (1995) showed that interstellar velocities for many lines of sight to nearby 


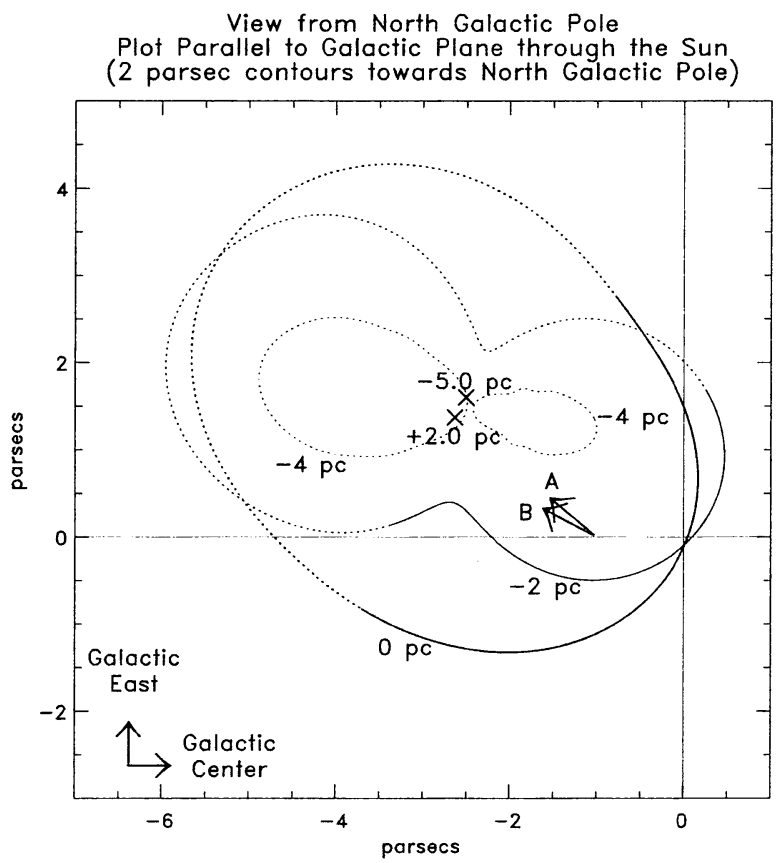

Figure 1. View of the LIC from the north Galactic pole. The Sun is located at $(0,0)$. The $\mathrm{x}$ symbols mark the northernmost and southernmost points of the LIC. The solid portions of the contours indicate where the model is well constrained. Arrow A indicates the direction from the center of the Sco-Cen association $\left(l=320^{\circ}, b=+15^{\circ}\right)$. Arrow $\mathrm{B}$ is the direction of the flow vector within the LIC in the local standard of rest $\left(l=331.9^{\circ}, b=+4.6^{\circ}\right)$. From Redfield \& Linsky (2000).

stars are consistent with a single velocity vector of the Local Interstellar Cloud (LIC). Furthermore, the Sun must be located inside the LIC as the flow vector of $\mathrm{H} \mathrm{I}$ in the heliosphere is consistent with the LIC flow vector (Witte et al. 1993). Linsky et al. (2000) and Redfield \& Linsky (2000) computed a model for the LIC based on values of $N_{\mathrm{HI}}$ at the projected LIC velocity for 16 lines of sight studied by HST, 3 lines of sight for which the analysis of EUVE spectra measured the total $N_{\mathrm{HI}}$, and 13 lines of sight with high resolution spectra in the Ca II $3933 \AA$ line. Figure 1 shows this model as viewed from the North Galactic Pole. We find that the Sun is located just inside the LIC $(<0.05 \mathrm{pc}$ from the edge) and that the flow inside the LIC is in nearly the same direction as the flow from the Sco-Cen Association, suggesting that the LIC is a warm condensation in the hot expanding gas produced by stellar winds and supernovae in Sco-Cen.

Figure 2 shows contours of $N_{\mathrm{HI}}$ in the LIC projected on to the sky in Galactic coordinates. The maximum column density $N_{\mathrm{HI}}=2.1 \times 10^{18} \mathrm{~cm}^{-2}$ is approximately in the direction of the Pleiades. Also shown are the stars used in creating the model. There is very little LIC absorption toward the Galactic Center or the North Galactic Pole. This map and a tool for computing $N_{\mathrm{HI}}$ and 


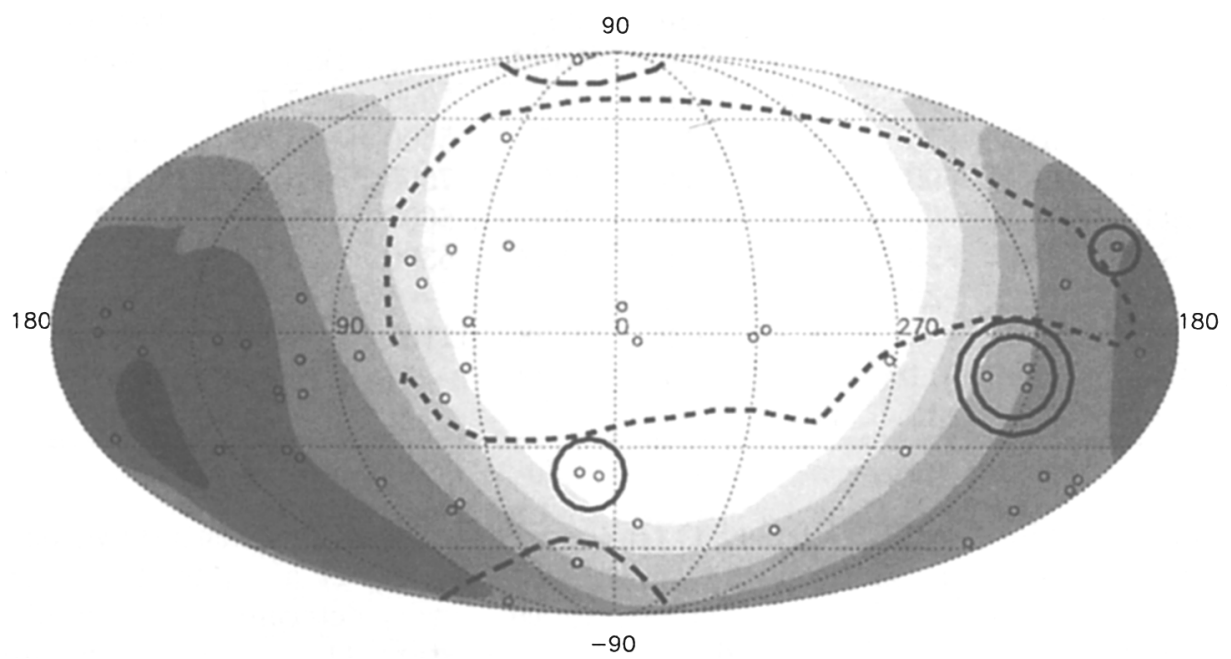

Figure 2. Projection of the LIC model on the sky in Galactic coordinates. The shadings indicate the values of $N_{\mathrm{HI}}$ in units of $10^{18} \mathrm{~cm}^{-2}$ from the Sun to the edge of the LIC. From darkest to lightest, the shadings designate $>2.0,1.0-2.0,0.5-1.0,0.25-0.5,0.10-0.25,0.05-$ 0.10 , and $<0.05$ in these units. The large dashed contour indicates the location of the G cloud, and the smaller dashed contours indicate the locations of the North Galactic Pole and South Galactic Pole clouds. Solid lines indicate the contours of four other clouds (see Table 1).

flow velocity along any line of sight through the LIC can be found in our web site http://casa.colorado.edu/ sredfiel/ColoradoLIC.html.

Several velocity components are typically observed in high resolution spectra even for short lines of sight. In many cases we can identify other warm clouds from several lines of sight that show similar velocities. Figure 2 shows approximate contours for these other clouds. The largest is the G (or Galactic Center) cloud originally identified by Lallement and Bertin (1992). There are also clouds near the North Galactic Pole (NGP) and South Galactic Pole (SGP). Other smaller clouds are identified by their heliocentric radial velocities. Table 1 lists the directions and distances $(d)$ of the inferred cloud centers, cloud thicknesses $\left(\Delta d\right.$ in pc and $\left.N_{\mathrm{HI}}\right), T, \xi$, and logarithmic depletion of magnesium, $\mathrm{D}(\mathrm{Mg})$. Except for the LIC and G clouds, these parameters are first approximations based on only a few lines of sight. The cloud thicknesses are estimated from the largest value of $N_{\mathrm{HI}}$ for the cloud and the assumption that $n_{\mathrm{HI}}=0.10$ $\mathrm{cm}^{-3}$. The volume of the LIC is about $93 \mathrm{pc}^{3}$ and its mass is about $0.32 \mathrm{M}_{\odot}$. The velocity vectors for the G, NGP, and SGP clouds are similar to that of the LIC and these clouds may also be warm condensations in the Sco-Cen outflow.

The electron density in the LIC, derived from the ratio of C II column densities from the ground state $(1335 \AA)$ and the first excited state $(1336 \AA)$, is about $n_{e}=0.11 \mathrm{~cm}^{-3}$ for the lines of sight to Capella (Wood \& Linsky 1997) 
Table 1. Properties of nearby warm clouds in the local ISM.

\begin{tabular}{|c|c|c|c|c|c|c|c|c|}
\hline Cloud & \multicolumn{2}{|c|}{ Center } & $d$ & $\Delta d$ & $N_{\mathrm{HI}}$ & $T$ & $\xi$ & $\mathrm{D}(\mathrm{Mg})$ \\
\hline Name & $l$ & $b$ & (pc) & (pc) & $\left(10^{18}\right)$ & (K) & $\left(\mathrm{km} \mathrm{s}^{-1}\right)$ & $(\log )$ \\
\hline LIC & 149 & -27 & 2.9 & 6.8 & 2.1 & 7000 & 1.7 & -1.1 \\
\hline G & 315 & +25 & 1.15 & 2.3 & 0.7 & 5600 & 1.7 & -0.5 \\
\hline NGP & - & +90 & 5.6 & 2.9 & 0.9 & 7500 & $<1.5$ & -1.4 \\
\hline SGP & - & -90 & 5.6 & 11.0 & 3.4 & 6600 & $<2.0$ & +0.3 \\
\hline+32 & 191 & +23 & 5.1 & 2.0 & 0.63 & 6300 & 1.0 & -1.3 \\
\hline-22 & 010 & -38 & 5.0 & 6.5 & 2.0 & - & - & - \\
\hline+12 & 189 & -50 & 5.2 & 0.9 & 0.28 & 11100 & 0.7 & -1.4 \\
\hline $\mathrm{BC}$ & 230 & -11 & 1.3 & 0.7 & 0.22 & - & - & $(-1.0)$ \\
\hline $\mathrm{CMa}$ & 233 & -12 & 65. & - & - & - & - & - \\
\hline
\end{tabular}

and the white dwarf REJ1032+532 (Holberg et al. 1999). The neutral hydrogen number density is more difficult to estimate. Linsky et al. (2000) estimate $n_{\mathrm{HI}}=0.10 \mathrm{~cm}^{-3}$ as this is the largest average density determined by assuming that the LIC fills the line of sight to the nearest stars. Other estimates for $n_{\mathrm{HI}}$ lie in the range $0.15-0.20 \mathrm{~cm}^{-3}$ as inferred from space observations of scattered solar L $\alpha$ photons by heliospheric H I (e.g., Quémerais et al. 1995).

Acknowledgments. This work is supported by NASA grants S-56500-D and GO-07262.01-99A to the University of Colorado and NIST.

\section{References}

Baranov, V.B. \& Malama, Y.G. 1995, JGR, 100, 14755

Holberg, J.B., et al. 1999, ApJ, 517, 841

Lallement, R., \& Bertin, P. 1992, A\&A, 266, 479

Lallement, R., Ferlet, R., Lagrange, A.M., Lemoine, M., \& Vidal-Madjar, A., 1995, A\&A, 304, 461

Linsky, J.L. 1998, Space Sci. Rev., 84, 285

Linsky, J.L., Diplas, A., Wood, B.E., Brown, A., Ayres, T.R., \& Savage, B.D. 1995, ApJ, 451, 335

Linsky, J.l., Redfield, S., Wood, B.E., \& Piskunov, N., 2000, ApJ, 528, 756

Quémerais, E., et al. 1995, A\&A, 299, 249

Redfield, S., \& Linsky, J.L. 2000, ApJ, 534, 825

Witte, M., Rosenbauer, H., Banaszkiewicz, M., \& Fahr, H., 1993, Adv. Space Res., 13, 121

Wood, B.E., Alexander, W.R., \& Linsky, J.L. 1996, ApJ, 470, 1157

Wood, B.E., \& Linsky 1997, ApJ, 474, L39

Wood, B.E., Linsky, J.L., \& Zank, G.P. 2000, ApJ, 537, 304

Wood, B.E., Müller, H.-R., \& Zank, G.P. 2000, ApJ, to appear Oct 10

Zank, G.P. 1999, Space Sci. Rev., 89, 413 